\title{
Fitting of precipitation in 49 European capitals from 1901 to 1998 using random walk
}

\author{
Shaomin $\mathrm{Yan}^{1}$, Guang $\mathrm{Wu}^{1,2^{\star}}$ \\ ${ }^{1}$ State Key Laboratory of Non-Food Biomass Enzyme Technology, National Engineering Research Center for Non-Food Biorefinery, \\ Guangxi Key Laboratory of Biorefinery, Guangxi Academy of Sciences, Nanning, China \\ ${ }^{2}$ DreamSciTech Consulting, Shenzhen, Guangdong, China; "corresponding author: hongguanglishibahao@yahoo.com
}

Received 15 March 2011; revised 5 April 2011; accepted 15 April 2011.

\begin{abstract}
Mathematical modeling of precipitation is an important step to understand the precipitation patterns, and paves the way to possibly predict the precipitation. In this study, we attempt to use the random walk model to fit the annual precipitation in 49 European capitals from 1901 to 1998. At first, we used the simplest random walk model to fit the precipitation walk, which is the conversion of recorded precipitations into \pm 1 format, and then we used a more complex random walk model to fit the recorded precipitations. The results show that the random walk models can fit both precipitation walk and recorded precipitation. Thus this study provides a model to describe the precipitation patterns during this period in these cities.
\end{abstract}

Keywords: European Capital; Fitting; Precipitation; Random Walk

\section{INTRODUCTION}

Mathematical description of precipitation is important, not only because it can help up better understand the precipitation pattern, but also it can provide a tool to possibly predict the precipitation. However, a mathematical model is not so easy to build because the precipitation is related to enormous factors, which lead to the difficulty to use a deterministic model to describe the precipitation. Technologically, the deterministic model is generally based on the cause-consequence relationship, which can be modeled as differential equations. As many cause-consequence relationships coexist in precipitation process, one would have great difficulty to separate various cause-consequence relationships notably because we cannot conduct a control experiment to determine each cause-consequence relationship. Still we have many unknown factors, which are not possible to include in a deterministic model. Computationally, a deterministic model with many factors would have great difficulty to fit the recorded data.

In this context, we may consider alternative models, such as stochastic models, of which the random walk is an important model to describe natural phenomena, for examples, stock pattern [1] and temperature change [2, 3]. Thus an interesting question raised here is whether the random walk model can describe the precipitation along with other powerful deterministic models? Practically, we cannot find any clearly visible patterns in yearto-year precipitations if we scrutinize the recorded precipitations over year in certain geographic location. The non-patterned precipitations would provide us with the opportunity to use the random walk model to fit.

To answer this question, we use the random walk model to fit the annual precipitations in 49 European capitals from 1901 to $\mathrm{m} 1998$ in this study.

\section{MATERIALS AND METHODS}

\subsection{Data}

The precipitations recorded in these 49 European capitals from 1901 to 1998 were obtained from the website of Oak Ridge National Laboratory [4], and their latitudes and longitudes were determined using Get Lat Lon [5] in order to define the precise precipitations according to the $0.5^{\circ}$ by $0.5^{\circ}$ latitude and longitude grid-box.

\subsection{Precipitation Walk}

We use the simplest random walk model, which starts at zero and moves by \pm 1 with equal probability at each step [6]. As the precipitation is in decimal format, thus we convert the precipitation into \pm 1 format as precipitation walk. Technically, when the precipitation at certain year is higher than its previous year, we classify it as 1 , otherwise we classify it as -1 , and then we add them together as the random walk does. 


\subsection{Generating Random Walk}

We use the SigmaPlot [7] with different seeds to generate random sequences ranged from -1 to 1 , and then we classify a random number as 1 if it is larger than its previous one and as -1 if it is smaller than its previous one. Thereafter we add the classified \pm 1 as random walk.

\subsection{Searching for Seed}

To the best of our knowledge, there is no algorithm available to find the right seed, which produces the best fit between random walk and observed data. However, this is not a problem with current computational technique, because we can simply search all the seeds in searching space and compare their outcomes.

\subsection{Fitting Recorded Precipitation}

Hereafter, we use a more complicated random walk model [8] to fit the recorded precipitation, which is in decimal format. In plain words, the simplest random walk comes from tossing of double-sided coin, while this random walk could be regarded as tossing of dice, which can be not only six-sided but as many as the decimal data. In this way, we generate random numbers, and add them to construct the random walk, and the fitting is again to search the best seed that generates best fit.

\subsection{Comparison}

For determining the best seed, we compare the least squared errors between precipitation walk and random walk, and between recorded precipitation and random precipitation generated from different seeds.

\section{RESULTS AND DISCUSSION}

Table 1 shows how we construct a precipitation walk and its corresponding random walk. For the precipitation walk, we have the follows: 1) the starting point is the annual precipitation in 1901, $847 \mathrm{~mm}$ (cell 2, column 2), and this starting point corresponds zero in sense of precipitation walk (cell 2, column 4); 2) the annual precipitation in 1902 is $737 \mathrm{~mm}$ (cell 3, column 2), which is smaller than the first one, $847 \mathrm{~mm}$ (cell 2, column 2), so we assign -1 as precipitation step (cell 3, column 3$), 3$ ) the precipitation walk is $-1(0+(-1))$ (cell 3 , column 4$)$, and 4$)$ the similar computation is applied to all the data in columns 2, 3, and 4 .

For the random walk, we have the follows: 1) a good seed we found is 6.98078 , and this seed generates a series of random numbers (column 5), 2) the first random number, 0.36795 (cell 2, column 5), is considered as the starting point corresponding to 0 in random walk (cell 2 , column 7), 3) the second random number, -0.74132 (cell 3 , column 5), is smaller than the first random number, 0.36795 (cell 2, column 5), so we assign -1 (cell 3, column 6), 4) the random walk is $-1(0+(-1))$ (cell 3 , column 7), and 5) the similar procedure is applied to all the data in columns 5,6 , and 7 . In the same manner, we construct the precipitation walk and random walk.

The figures in the left side of Figure 1 show the fittings of precipitation walk in 7 European capitals using random walk model. As can be seen, the curve generated by random walk generally passes through the precipitation walk. Theoretically, the chance for a completely perfect fitting of precipitation walk is an extremely rare event. In our case, there are 98 annual precipitations, thus the completely perfect fitting has the chance of $(1 / 2)^{98}$ theoretically, which is extremely small. Clearly this probability is very difficult to achieve in limited time because the space of our search is limited to one million of seed. So the fitting results in the left side of Figure 1 suggest that a good seed can be relatively easily found, thus we consider that the random walk can describe the precipitation walk, although we cannot compare our results with other results because the other models do not set an equal-sized step.

Actually we can view the precipitation walk, which is the conversion from its annual precipitation, as the trend of recorded precipitation. This is so because this trend answers the very basic question of whether the precipitation at certain year is larger (1) or smaller $(-1)$ than its previous year.

Yet, the cities in Figure 1 cross the whole Europe, thus there would be uncountable factors affecting the precipitations, but the random walk still can fit them. This furthermore suggests that the random walk can describe the precipitation patterns in terms of precipitation walk.

Table 2 shows how we use a random walk model to fit the recorded annual precipitation, here we only need to construct the random precipitation: 1) the starting point is the first recorded annual precipitation, which is $847 \mathrm{~mm}$ (cell 2 in column 2 and column 4), 2) the seed for Table 2 is $1.31923,3$ ) the first random number generated by the seed is 64.17878 (cell 3 , column 3 ), 4) we add this value to the previous precipitation datum (847) resulting in $911.17878 \mathrm{~mm}$ (cell 3, column 4), and 5) along this procedure, we get the random precipitation in column 4.

The figures in the right side of Figure 1 display the fittings of recorded precipitation with random precipitation in 7 European capitals. In these figures, the precipitation demonstrates very remarkable fluctuations along the time course, which do not show any clear sign of visible pattern. This is the basis for conducting random- 


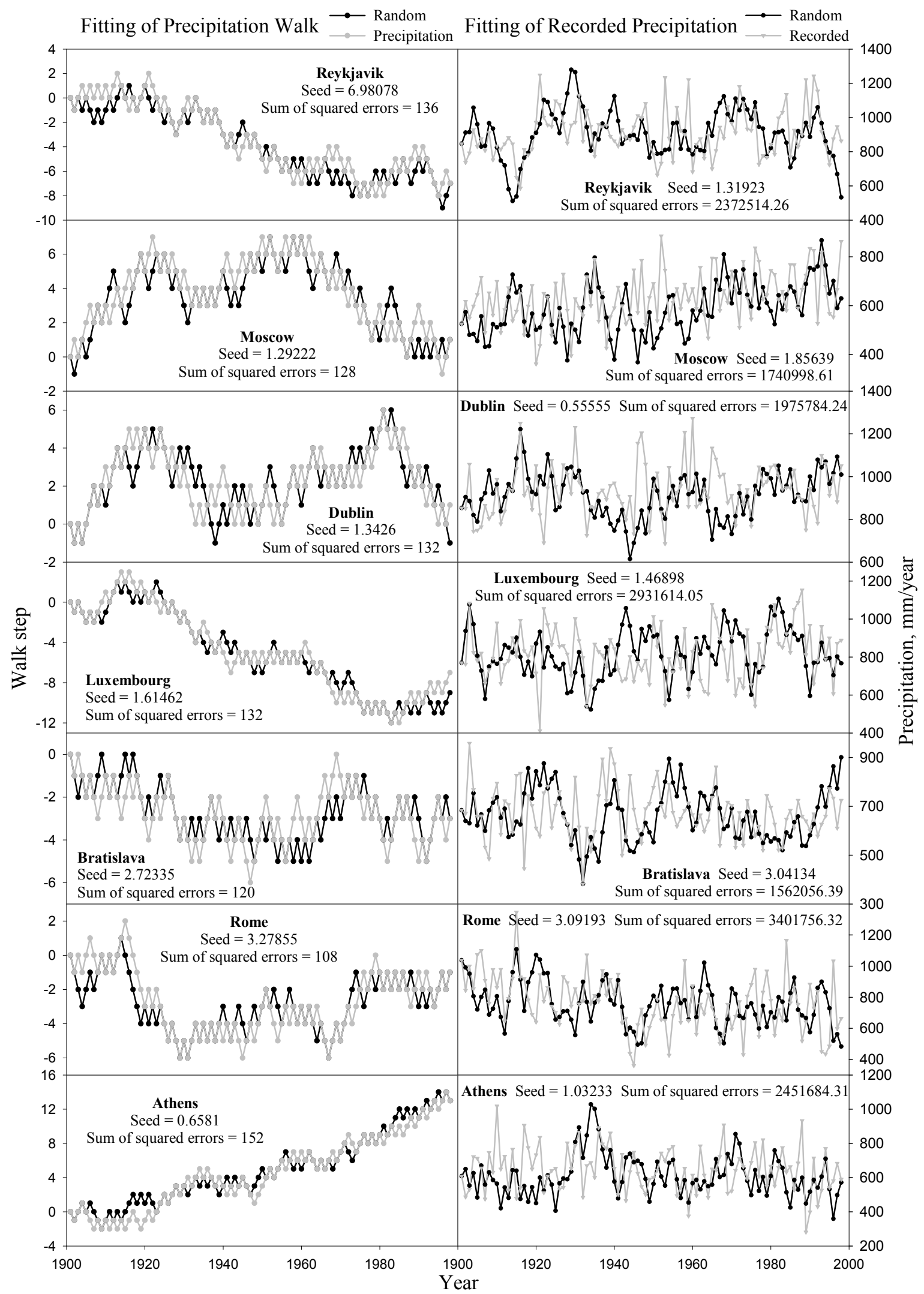

Figure 1. Comparison of precipitation walk with random walk and of recorded precipitation with random precipitation in 7 European capitals from 1901 to 1998. 
Table 1. Conversion of recorded precipitation into precipitation walk and generation of random walk for precipitation in Reykjavík from 1901 to 1998.

\begin{tabular}{|c|c|c|c|c|c|c|}
\hline Year & $\begin{array}{l}\text { Precipitation } \\
\mathrm{mm} / \text { year }\end{array}$ & $\begin{array}{l}\text { Precipitation } \\
\text { Step }\end{array}$ & $\begin{array}{l}\text { Precipitation } \\
\text { Walk }\end{array}$ & $\begin{array}{c}\text { Generated Random } \\
\text { Number }\end{array}$ & Random Step & Random Walk \\
\hline 1901 & 847 & & 0 & 0.36795 & & 0 \\
\hline 1902 & 737 & -1 & -1 & -0.74132 & -1 & -1 \\
\hline 1903 & 793 & 1 & 0 & 0.03941 & 1 & 0 \\
\hline 1904 & 929 & 1 & 1 & -0.05685 & -1 & -1 \\
\hline 1905 & 830 & -1 & 0 & 0.27137 & 1 & 0 \\
\hline 1906 & 876 & 1 & 1 & -0.54282 & -1 & -1 \\
\hline 1907 & 807 & -1 & 0 & -0.85436 & -1 & -2 \\
\hline 1908 & 911 & 1 & 1 & 0.02900 & 1 & -1 \\
\hline 1909 & 752 & -1 & 0 & -0.31867 & -1 & -2 \\
\hline 1910 & 833 & 1 & 1 & 0.67880 & 1 & -1 \\
\hline$\ldots$ & $\ldots$ & ... & $\ldots$ & $\ldots$ & $\ldots$ & $\ldots$ \\
\hline 1991 & 1243 & 1 & -4 & -0.03583 & -1 & -6 \\
\hline 1992 & 1155 & -1 & -5 & 0.53701 & 1 & -5 \\
\hline 1993 & 918 & -1 & -6 & -0.31605 & -1 & -6 \\
\hline 1994 & 841 & -1 & -7 & -0.44354 & -1 & -7 \\
\hline 1995 & 723 & -1 & -8 & -0.63220 & -1 & -8 \\
\hline 1996 & 875 & 1 & -7 & -0.87762 & -1 & -9 \\
\hline 1997 & 947 & 1 & -6 & -0.80406 & 1 & -8 \\
\hline 1998 & 863 & -1 & -7 & -0.10839 & 1 & -7 \\
\hline
\end{tabular}

The seed for generation of random numbers is 6.98078 using SigmaPlot.

Table 2. Generation of recorded precipitation into random precipitation in Reykjavík from 1901 to 1998.

\begin{tabular}{cccc}
\hline Year & $\begin{array}{c}\text { Recorded Precipitation } \\
\text { mm/year }\end{array}$ & $\begin{array}{c}\text { Generated Random Number } \\
\text { mm/year }\end{array}$ & 847.00000 \\
1901 & 847 & 611.17878 & 914.14245 \\
1902 & 737 & 2.96367 & 1057.37302 \\
1903 & 793 & 143.23057 & 959.93507 \\
1904 & 929 & -97.43795 & 828.97585 \\
1905 & 830 & -130.95922 & 833.70785 \\
1906 & 876 & 4.73200 & 966.68164 \\
1907 & 807 & 132.97379 & 934.67541 \\
1908 & 911 & -32.00623 & 824.39724 \\
1909 & 752 & -110.27817 & $\ldots$ \\
1910 & 833 & $\ldots$ & 998.44105 \\
1991 & $\ldots$ & 112.18813 & 1059.02499 \\
1992 & 1243 & 60.58394 & 966.10101 \\
1993 & 1155 & -92.92398 & 860.24102 \\
1994 & 918 & -105.85999 & 795.32882 \\
1995 & 841 & -64.91220 & 775.02212 \\
1996 & 723 & -20.30670 & 668.90237 \\
1998 & 875 & -106.11975 & \\
532.79860 & \\
& 947 & -136.10377 & \\
\end{tabular}

The seed for generation of random numbers is 1.31923 using SigmaPlot.

walk to fit the precipitation data. As can be seen, the random model did generate the curves similar to the recorded annual precipitations. This is particularly important, because the recorded annual precipitation presents a very difficult pattern to be fit by any other models.

Due to the limitation of space, we did not present our fittings for all 49 European capitals, not only because seven European capitals in Figure 1 come from almost each corner and the central of Europe, but also because one can make graphic observation with the seeds listed in Table 3 using SigmaPlot to generate fitted curves to compare with recorded ones.

The data used in our study spanned for almost a cen- tury. With 98 annual precipitations, we would find a good estimate because the seed is the only model parameters for random walk. However, the uncertainty would increase if we use a more complicated model, which contains more model parameters.

Actually, the literature search does not show many studies using random walk to fit the historical data. There could be several reasons for the lack of use of random walk. 1) Our fitting technique mainly concen trated in deterministic models in the past while the fitting using random walk is largely ignored. 2) Although computational technique advanced significantly, to fully try each possibility of tossing of coin is still very diffi- 
Table 3. Model parameters (seeds) and fitted results for fitting precipitation change in 49 European capitals from 1901 to 1998 using random walk model.

\begin{tabular}{|c|c|c|c|c|c|}
\hline \multirow[t]{2}{*}{ State } & \multirow[t]{2}{*}{ Capital } & \multicolumn{2}{|c|}{ Fitting of Precipitation Walk } & \multicolumn{2}{|c|}{ Fitting of Recorded Precipitation } \\
\hline & & Seed & Sum of Squared errors & Seed & Sum of Squared errors \\
\hline Albania & Tirana & 5.89746 & 160 & 3.09458 & 4480260.88 \\
\hline Andorra & Andorra la Vella & 0.76994 & 120 & 2.04962 & 3762379.49 \\
\hline Armenia & Yerevan & 5.11706 & 140 & 1.03292 & 1654018.73 \\
\hline Austria & Vienna & 5.29984 & 120 & 6.26795 & 1854605.22 \\
\hline Azerbaijan & Baku & 1.83503 & 144 & 1.17243 & 580310.28 \\
\hline Belarus & Minsk & 3.91196 & 124 & 6.87272 & 1136251.86 \\
\hline Belgium & Brussels & 1.32931 & 128 & 3.79933 & 2793049.95 \\
\hline $\begin{array}{c}\text { Bosnia and Her- } \\
\text { zegovina }\end{array}$ & Sarajevo & 2.34269 & 120 & 3.89254 & 4488970.73 \\
\hline Bulgaria & Sofia & 1.47405 & 136 & 3.50057 & 1574839.07 \\
\hline Croatia & Zagreb & 4.75272 & 132 & 1.62778 & 4530028.69 \\
\hline Cyprus & Nicosia & 0.96966 & 124 & 1.68592 & 1959226.37 \\
\hline Czech Republic & Prague & 2.41744 & 156 & 1.75624 & 1180350.59 \\
\hline Denmark & Copenhagen & 2.67161 & 136 & 1.75624 & 979500.29 \\
\hline Estonia & Tallinn & 3.68127 & 112 & 0.75372 & 1719360.48 \\
\hline Finland & Helsinki & 1.17561 & 119 & 3.88438 & 1601835.51 \\
\hline France & Paris & 0.71257 & 137 & 0.01458 & 1671903.49 \\
\hline Georgia & Tbilisi & 4.04021 & 143 & 1.03292 & 1876296.69 \\
\hline Germany & Berlin & 1.53962 & 100 & 8.04385 & 1267406.51 \\
\hline Greece & Athens & 0.65810 & 152 & 1.03233 & 2451684.31 \\
\hline Hungary & Budapest & 2.24208 & 124 & 2.26215 & 3274954.55 \\
\hline Iceland & Reykjavík & 6.98078 & 136 & 1.31923 & 2372514.26 \\
\hline Ireland & Dublin & 1.34260 & 132 & 0.55555 & 1975784.24 \\
\hline Italy & Rome & 3.27855 & 108 & 3.09193 & 3401756.32 \\
\hline Latvia & Riga & 1.63481 & 120 & 2.23707 & 982200.71 \\
\hline Liechtenstein & Vaduz & 5.32749 & 140 & 7.48196 & 6544143.92 \\
\hline Lithuania & Vilnius & 2.64178 & 128 & 3.80609 & 1817826.21 \\
\hline Luxembourg & Luxembourg & 1.61462 & 132 & 1.46898 & 2931614.05 \\
\hline $\begin{array}{l}\text { Republic of Ma- } \\
\text { cedonia }\end{array}$ & Skopje & 2.61614 & 132 & 2.21326 & 2413733.26 \\
\hline Malta & Valletta & 2.29461 & 124 & 1.03233 & 1683481.23 \\
\hline Moldova & Chişinău & 1.02884 & 116 & 0.53868 & 1976370.09 \\
\hline Monaco & Monaco & 0.07616 & 128 & 3.50057 & 5805776.86 \\
\hline Montenegro & Podgorica & 0.64299 & 184 & 3.89254 & 7373847.85 \\
\hline Netherlands & Amsterdam & 1.11756 & 96 & 1.8384 & 2103720.16 \\
\hline Norway & Oslo & 5.15239 & 128 & 0.49518 & 2762908.96 \\
\hline Poland & Warsaw & 6.38046 & 136 & 3.73365 & 1099078.36 \\
\hline Portugal & Lisbon & 5.62135 & 128 & 1.94803 & 3988957.69 \\
\hline Romania & Bucharest & 2.96749 & 124 & 2.82657 & 1634074.60 \\
\hline Russia & Moscow & 1.29222 & 128 & 1.85639 & 1740998.61 \\
\hline San Marino & San Marino & 2.86281 & 160 & 1.65197 & 2562453.91 \\
\hline Serbia & Belgrade & 5.30232 & 145 & 2.82657 & 2285551.27 \\
\hline Slovakia & Bratislava & 2.72335 & 120 & 3.04134 & 1562056.39 \\
\hline Slovenia & Ljubljana & 1.07884 & 136 & 3.89254 & 5247413.75 \\
\hline Spain & Madrid & 1.81411 & 132 & 0.56663 & 1181419.10 \\
\hline Sweden & Stockholm & 9.22779 & 120 & 3.24016 & 834669.37 \\
\hline Switzerland & Bern & 6.31144 & 152 & 1.03233 & 7547487.27 \\
\hline Turkey & Ankara & 2.57067 & 147 & 1.6555 & 633833.41 \\
\hline Ukraine & Kiev & 1.24473 & 124 & 6.08046 & 1628394.92 \\
\hline United Kingdom & London & 9.43653 & 136 & 3.79933 & 1801453.26 \\
\hline Vatican City & Vatican City & 3.28152 & 108 & 3.09193 & 3401756.32 \\
\hline
\end{tabular}

cult. For example, the complete fitting of 98 annual precipitations require $316,912,650,057,057,350,374$, $175,801,344$ trials $(1 / 2)^{98}$, which is a very difficult task because not only the time for computation is very considerable but also it is doubt whether the current MonteCarlo algorithm could generate so many different seeds. We found the trend within 1,000,000 trials, so the probability is extremely small $(1 / 2)^{91}$, which does suggest the precipitation trend.
On the other hand, it is not clear whether we can satisfyingly use the seed, which fits the first 49-year precipitations, to predict the second 49-year precipitations, not only because this research area is far less studied but also it is difficult for any deterministic model to use the parameters obtained from fitting of first half data to predict the second half data. Moreover, the focus in this study is to see whether a random walk can fit the recorded precipitation, which should be the first step for 
the predictions that need many studies in the future.

Currently, no results are available from other models on fitting the precipitation of these cities for comparison. However, our results are encouraging because the random walk model provides a way to describe the precipitation pattern.

In conclusion, the results show that the random walk model can describe the annual precipitation pattern.

\section{ACKNOWLEDGEMENTS}

This study was partly supported by Guangxi Science Foundation (07-109-001A, 08-115-011, 09322001, 10-046-06 11-031-11, 2010GXNSFF013003 and 2010GXNSFA 013046). The authors wish to thank Dr Hong Zhang at Biyee SciTech Inc., MA, USA for helpful discussion. The authors also wish to thank the Library of Guangxi Zhuang Autonomous Region for purchasing the book, An Introduction to Probability Theory and Its Applications.

\section{REFERENCES}

[1] Yan, S. and Wu, G. (2011) Fitting of SSEC index
(Shanghai Composite) from January 2000 to July 2010 using random walk model. Guangxi Sciences, 18, 92-96.

[2] Yan, S. and Wu, G. (2010) Modeling of global temperature change from 1850 to 2009 using random walk. $G u$ angxi Sciences, 17, 148-150.

[3] Yan, S. and Wu, G. (2010) Application of random walk model to fit temperature in 46 gamma world cities from 1901 to 1998. Natural Sciences, 2, 1425-1431. doi:10.4236/ns.2010.212174

[4] ORNL DAAC Data Holdings. (2010) http://daac.ornl.gov/holdings.html

[5] Willison, S. (2010) Get Lat Lon. http://www.getlatlon.com/

[6] Feller, W. (1968) An introduction to probability theory and its applications. Third Edition, Wiley, New York.

[7] SPSS Inc., SigmaPlot 2002 for Windows Version 8.02. (1986-2001).

[8] Borovkov, A. and Borovkov, K. (2008) Asymptotic analysis of random walks: Heavy-tailed distributions. Cambridge University Press, Cambridge. doi:10.1017/CBO9780511721397 